\title{
Anthropometric changes following aerobic and resistance training programs among HIV-seropositive female patients
}

\author{
Oluwaseun Kubeyinje $^{1}$ (D), Agharese Efe-Aigbovo ${ }^{2}$ (]) \\ ${ }^{1}$ Department of Physiotherapy, University of Benin Teaching Hospital, Benin City. ${ }^{2}$ Department of Human Kinetics and Sports \\ Science, University of Benin, Benin City.
}

\begin{abstract}
This study was designed to assess the changes in anthropometric parameters of HIVseropositive patients following 6-week aerobic and resistance exercise training. The pretest-posttest control-group design (experimental and control groups) was adopted. Sixty female HIV-seropositive patients in stage 1 and 2 of the disease registered in University of Benin Teaching Hospital participated in the study. They were randomly assigned into control $(n=19)$ and experimental groups $(n=25)$. The experimental group exercised for One hour, three times a week for six weeks while the control group continued with their regular medications and counseling. The baseline intensity of the exercise was $60 \%$ Heart Rate Reserve (HRR) and 60\% onerepetition maximum and it was progressed by $10 \%$ every 2 weeks. Anthropometric parameters were recorded at baseline and on completion of 6 weeks for both groups. Data were analyzed with inferential statistic (Analysis of variance) to test the hypotheses. The findings of this study showed that short duration exercise training did not result in significant changes in anthropometric parameters of body mass index, percentage body fat, visceral fat and waist to hip ratio. It was therefore recommended based on the findings of this study that health professionals should recommend exercise for female HIV-infected individuals due to the health benefits.
\end{abstract}

Keywords. Aerobic, anthropometry, HIV, resistance.

\section{Introduction}

The Human Immune-deficiency Virus/Acquired Immunodeficiency Syndrome (HIV/AIDS) epidemic is on the increase around the world. Despite the fact that significant efforts are being made nationally and internationally to diminish the transmission of HIV, it is still actively spreading in countries and it can be said to be a pandemic. However, the advent of Highly Active Anti- Retroviral Therapy (HAART) has significantly reduced the fatality of HIV infection. People Living with HIV/AIDS (PLWHA) who are on HAART have longer life expectancy making the disease now a chronic one. The chronicity of HIV/AIDS is not without associated effects on the quality of life of PLWHA as well as the side-effects of HAART.

Globally, 36.9 million (34.4-41.4 million) people were living with HIV at the end of 2014, but almost half $(46 \%)$ of them did not know their HIV status (World Health Organization [WHO], 2017). An estimated $0.8 \%$ of adults aged 15-49 years worldwide are living with HIV, although the burden of the epidemic continues to vary considerably between countries and regions. Sub-Saharan Africa remains most severely affected, with nearly 1 in every $20(4.8 \%)$ adults living with HIV and accounting for nearly $70 \%$ 
of the people living with HIV worldwide (WHO, 2017). In fact, Nigeria accounts for $9 \%$ of People Living with HIV/AIDS (PLWHA). Women constitute the fastest group of new HIV/AIDS diagnoses (Bokazhanova \& Rutherford, 2006). Recent statistics in Nigeria showed that women between the ages of 15 and 49 years are mostly affected by HIV/AIDS.

Physical activity and exercise have been reported in several studies to prevent chronic diseases and also help in improving the quality of life of persons diagnosed with chronic diseases such as diabetes mellitus, chronic obstructive airway diseases, cancers, depression etc. (American College of Sports Medicine [ACSM], 2010). Exercise has also been demonstrated by several studies to be beneficial to both the healthy and diseased population. Its benefits in the prevention of non-communicable diseases have been well documented in both developed and developing countries. A common practice among healthy and other clinical populations to help modify these risk factors is some form of aerobic exercise, resistance exercise, or a combination of both. It has been demonstrated that PLWHA could respond in a similar manner, which in turn may enhance life expectancy and/or quality of life. Exercise has the potential to ameliorate a range of side effects associated with HIV infection, as well as the cardio metabolic and morphological complications (i.e., mitochondrial dysfunction, inflammation, and oxidative stress) that may accompany HAART.

Aerobic Exercise (AE) can be used to optimize the level of physical fitness and maintain normal systemic body functions. AE is any activity that stimulates heart rate and increases breathing but not so much that one cannot sustain the activity for more than a few minutes. Resistance Training (RT) programmes are used to achieve many different goals, such as enhancement of athletic performance, reduction of the risk or rehabilita- tion of injury and improvement of systemic body functions. Thus, RT increases the concentration of various growth-promoting agents in the body that may contribute to these improved functions (ACSM, 2009).

Recent advances in research have shown that anthropometric measures now constitute the new vital signs of the 21st century, and therefore warrant urgent assessment if the tide of metabolic and other systemic conditions are to be turned. This is because the economic and social consequences of these conditions could be overwhelming especially in underdeveloped and developing countries. Anthropometry is the science that defines physical measures of a person's size, form, and functional capacities (Centre for Disease Control (CDC), 2018). Several components constitute anthropometric measures such as Body Mass Index (BMI), waistto-hip ratio and percentage body fat. BMI is considered globally the most used adiposity index in the body composition area (Beraldo et al., 2018). Body fat percentage evaluated by BIA is however considered the best double-indirect estimate of body fat and predictor of cardiovascular disease risk and presents a satisfactory performance but not exceeding the measures of central adiposity such as visceral fat and waist to hip ratio (Beraldo et al., 2018).

HIV-related disability has also been associated with decreased exercise capacity and impairment of patients' daily activities (Crystal et al., 2000), thus exercise training is a key strategy employed by patients with HIV or AIDS that is widely prescribed by rehabilitation professionals (Dudgeon et al., 2004). Adaptations to exercise are highly dependent on the specific type of training performed, however, there is no consensus regarding the modality and intensity of exercises that are more effective in patients with HIV, making it difficult to choose the best training for this population (Gomes-Neto et al., 2013). This presents a major obstacle in clinical 
practice. A better understanding of the effectiveness and safety of exercise will enable people living with HIV and their health care providers to practice effective and appropriate exercise prescription. In a review carried out by GomesNeto et al. (2013), it was concluded that considerable evidence currently exists to support a role for different types of exercise in the management of HIV-infected patients, but the evidence of this is sparse in Nigeria; particularly in the South-South geopolitical zone of Nigeria. In addition, health-care in Nigeria for PLWHA is mostly paid out of pocket and where there is health insurance, the referral for exercise therapy at physiotherapy clinics is usually for three weeks renewable possibly once. Therefore, this limits the duration of exercise training for this special group of patients except the individual pays out of pocket.

Thus, this study was aimed at finding out if short-duration (6 weeks) aerobic and resistance training programmes will alter the anthropometric characteristics of female HIVseropositive patients. It was therefore hypothesized that there would be no significant difference in the anthropometric characteristics (BMI, waist-to-hip ratio, percentage body fat and visceral fat) of female HIV-seropositive patients prior to and following a 6-week aerobic and resistance training programmes

\section{Methods}

\section{Research Design}

The design for this study was the pre-test/posttest experimental and control group design. This design is considered most appropriate because the participants were randomly assigned into control and experimental groups, subjected to an initial test (pre-test) measurement of the dependent variables, the treatment group was administered a treatment (independent variables) and the dependent variables were measured again in line with Bhattacherjee's (2012) contention.

\section{Population, Sample and Sampling Technique}

Two hundred and seventy (276) HIVseropositive female patients were registered and attending the clinic at the University of Benin Teaching Hospital's President's Emergency Plan for AIDS Relief (PEPFAR), Benin-City, Nigeria. One hundred and fifty-eight (158) patients met the inclusion criteria thus making the population for this study. The participants selected were adult female aged 18 to 60 years, diagnosed to be stages 1 and 2 HIV infection, previous sedentary lifestyle, no comorbidities and not pregnant. A simple random sampling technique was used to select the participants. Balloting without replacement was used to select one-third (1/3) of the population for the study resulting in 52.7 . $10 \%$ of the sample size (5.3) was added to take care of attrition, thus the figure was rounded up to 60 . Thus, a total of sixty (60) participants were selected out of the population.

\section{Research Instruments}

The instrument that was used is an adaptation of ACSM (2009) exercise training protocol. The training protocol was as shown in Table 1.

Table 1

ACSM (2009) exercise training protocol.

\begin{tabular}{llll}
\hline Exercises & Set & Repetition & $\begin{array}{l}\text { Rest between } \\
\text { sets }\end{array}$ \\
\hline Bicycle ergometer & $1^{*}$ & $(60 \% \mathrm{HRmax})$ & \\
Dumbbells & $3^{*}$ & $10(60 \% 1 \mathrm{RM})$ & 30 seconds \\
Ankle weight & $3^{*}$ & $10(60 \% 1 \mathrm{RM})$ & 30 seconds \\
\hline
\end{tabular}

\section{Method of Data Collection}

An ethical approval to conduct this study was received from the Research Ethics Committee of the University of Benin Teaching Hospital, 
Nigeria to ensure that the research was conducted in accordance to Helsinki declaration. Prior to the exercise programmes, a detailed explanation of the test, training programmes and; the objectives and intricacies of the study was provided to the participants and then the participants signed a participant's informed consent form before participating in this study. Thereafter, the participants were made to undergo a 6-week ( $11^{\text {th }}$ June to $20^{\text {th }}$ July, 2019) combined aerobic and resistance exercise training programmes of a frequency of 3 times per week (Monday, Wednesday, and Friday) with each session lasting for a total of 60 minutes. 10 minutes warm up, 20 minutes cycling on the bicycle ergometer at $60 \%$ HRmax, 20 minutes resistance exercise with dumbbells and ankle weights at $60 \%$ one-repetition maximum (60\% 1RM) and 10 minutes cool down. Progression of intensity was done every two weeks.

Physicians (Infectious disease specialist) at the PEPFAR clinic of the hospital screened the participants for eligibility based on the inclusion/exclusion criteria and conducted a physical examination on each participant. Each participant in addition to the screening and physical examination filled a Physical Activity Readiness Questionnaire (PAR-Q).

\section{Aerobic Exercise Training}

This was carried out on a stationary bicycle ergometer for duration of 20 minutes at $60 \%$ of target heart rate due to the fact that individuals who are HIV-seropositive easily fatigue. The participants were instructed to sit upright on the bicycle ergometer, with the feet on the pedals. The participants were instructed to ride the bicycle as fast as he/she could for 15 minutes. The session ended with a 5-minute recovery and relaxation phase. In order to counter the effect of adaptation, the intensity of the exercise was increased by $10 \%$ after 3 weeks of training.

\section{Resistance Exercise Training}

Free weights in the form of dumbbells and ankle weights was used for upper and lower limbs' strengthening using $60 \%$ of 10 Repetition Maximum (RM). RM is the heaviest weight that can be successfully lifted 10 times before fatiguing. Each exercise was done at the dosage of 3 sets of 10 repetitions with 30 seconds rest between each set. The session ended with a 5minute recovery and relaxation phase. In order to counter the effect of adaptation, the intensity of the exercise was also increased by $10 \%$ after 3 weeks of training. Meanwhile, subjects in the control group were instructed to continue their normal routine and not participate in any formal exercise program for the duration of the 6-week study.

\section{Measurement of Anthropometric Parameters}

\section{BMI, body fat percentage and visceral fat}

The standing heights were measured using a stadiometer. Each participant was assessed while in good standing posture. The participant stood on the base platform barefooted with their back to the measuring rod. Bulky headpieces and accessories were removed. The participants were instructed to stand straight and tall with the head was held in the Frankfort plane. The sliding headpiece paddle was then adjusted and measurement was taken to the nearest $0.1 \mathrm{~cm}$. Three measurements were taken and the average was recorded.

The digital weighing scale was used to measure body weight. Participants were instructed to empty their pockets and remove shoes and any apparel that could interfere with weight measurements. Omron body fat monitor was used to measure the BMI, body fat percentage and visceral fat of the participants. The scale was switched on and the self-check and zero balance were allowed to occur before the participant steps on the scale. The scale was 
set to measure in kilogram. The height, age, and sex of the participant were then be inputted into the digital monitor. The participants were weighed without shoes and wearing only light indoor clothing. Sweaters, jackets, coats, hats, scarves, and shoulder bags were removed. The participant was directed to stand in the middle of the weighing surface covered. The arm piece of the digital monitor was held by the participant with the shoulder in $90^{\circ}$ flexion and elbow joint in full extension. They then stood on the scale looking straight ahead, relaxed and remained motionless without leaning on any object or the wall.

The digital scale then locked in the measurement value. The digital scale stops blinking to indicate the measurement is locked. The display of the participant's BMI, body fat percentage and visceral fat was then noted.

\section{Waist and hip circumference}

A standard flexible measuring tape to the nearest centimetre was used to measure the waist and the hip circumferences. The landmark for the waist circumference was the midpoint between the last rib and the iliac crest. The measurement was taken at the end of normal expiration as recommended by WHO (2008). The hip circumference was measured at the widest part between the anterior-superior iliac crest and the greater trochanter for the hip circumference.

\section{Table 2}

Socio-demographic characteristics of HIV-seropositive female patients.

\begin{tabular}{|c|c|c|c|c|}
\hline \multirow{2}{*}{ Variables } & \multicolumn{2}{|c|}{ Experimental $(n=25)$} & \multicolumn{2}{|c|}{ Control $(n=19)$} \\
\hline & Frequency & Percentage & Frequency & Percentage \\
\hline \multicolumn{5}{|l|}{ Age(years) } \\
\hline $35-39$ & 8 & 32.0 & 6 & 31.6 \\
\hline $45-49$ & 6 & 24.0 & 6 & 31.6 \\
\hline $50-54$ & 7 & 28.0 & 7 & 36.8 \\
\hline $55-59$ & 4 & 16.0 & 0 & 0.0 \\
\hline Mean \pm SD & \multicolumn{2}{|c|}{$45.96 \pm 7.60$} & \multicolumn{2}{|c|}{$46.00 \pm 7.18$} \\
\hline \multicolumn{5}{|l|}{ Marital Status } \\
\hline Single & 0 & 0.0 & 6 & 31.6 \\
\hline Married & 19 & 76.0 & 8 & 42.1 \\
\hline Widowed & 6 & 24.0 & 5 & 26.3 \\
\hline \multicolumn{5}{|l|}{ Educational Level } \\
\hline None & 2 & 8.0 & 0 & 0.0 \\
\hline Primary & 9 & 36.0 & 5 & 26.3 \\
\hline Secondary & 12 & 48.0 & 12 & 63.2 \\
\hline Tertiary & 2 & 8.0 & 2 & 10.5 \\
\hline \multicolumn{5}{|l|}{ Occupation } \\
\hline Civil servant & 4 & 16.0 & 2 & 10.5 \\
\hline Business & 21 & 84.0 & 15 & 78.9 \\
\hline Unemployed & 0 & 0.0 & 2 & 10.5 \\
\hline
\end{tabular}




\section{Method of Data Analysis}

The data obtained in this study approximated that of a normal distribution using Q-Q plot chart. Thus, inferential statistics of analysis of variance (ANOVA) was used to test the hypotheses. Where there were significances, Tukey's LSD Post-hoc analysis was used to identify the source of significant difference. Statistical significance was set as p-value of $<0.05$.

\section{Results}

Table 2 presents the socio-demographic data (age, marital status, educational level and occupation) of the participants. The age of the experimental group was $45.96 \pm 7.60$ and the control group was $46.00 \pm 7.18$. In both groups, majority were married $(76 \%$ and $42.1 \%$ respectively). Most of the participants in both groups had secondary education as the highest level of education ( $48 \%$ and $63.2 \%$ respectively). Also, most of the participants in both groups were self-employed in business $(84 \%$ and $78.9 \%$ respectively).

Table 3 presents the anthropometric characteristics of the participants in the experimental and control groups before and after 6 weeks of exercise training.

Table 3

Anthropometric characteristics of HIV-seropositive female patients (Mean \pm SD).

\begin{tabular}{lcc}
\hline Variables & Experimental & Control \\
\hline Height & $159.74 \pm 10.09$ & $162.66 \pm 2.09$ \\
Pre Weight & $65.97 \pm 14.92$ & $67.07 \pm 18.54$ \\
Pre BMI & $25.78 \pm 4.11$ & $25.44 \pm 7.34$ \\
Post Weight & $65.98 \pm 14.88$ & $67.92 \pm 18.59$ \\
Post BMI & $25.70 \pm 4.12$ & $25.77 \pm 7.35$ \\
\hline
\end{tabular}

Table 4

ANOVA Analysis of the anthropometric parameters of HIV-seropositive female patients prior to and following a 6-week exercise training programme.

\begin{tabular}{|c|c|c|c|c|c|c|}
\hline Variables & & Sum of Squares & df & Mean Square & $\mathrm{F}$ & $p$ \\
\hline \multirow{3}{*}{ BMI } & Between Groups & 1.500 & 3 & .500 & .015 & .997 \\
\hline & Within Groups & 2753.508 & 84 & 32.780 & & \\
\hline & Total & 2755.008 & 87 & & & \\
\hline \multirow{3}{*}{ Body Fat } & Between Groups & 54.440 & 3 & 18.147 & .208 & .891 \\
\hline & Within Groups & 7340.716 & 84 & 87.389 & & \\
\hline & Total & 7395.156 & 87 & & & \\
\hline \multirow{3}{*}{ Visceral Fat } & Between Groups & 13.292 & 3 & 4.431 & .626 & .600 \\
\hline & Within Groups & 594.152 & 84 & 7.073 & & \\
\hline & Total & 607.443 & 87 & & & \\
\hline \multirow{3}{*}{ Waist to Hip Ratio } & Between Groups & .003 & 3 & .001 & .539 & .657 \\
\hline & Within Groups & .133 & 84 & .002 & & \\
\hline & Total & .136 & 87 & & & \\
\hline
\end{tabular}


Table 4 presents the results of the ANOVA of the anthropometric characteristics of the participants. It shows that there was no significant difference between the mean BMI, body fat, visceral fat and waist to hip ratio of the participants in the control and experimental groups prior to and following 6-week exercise training. Thus, the null hypotheses were accepted.

\section{Discussion}

The results of this study showed that six weeks of exercise training resulted in no significant difference in the BMI between the experimental and control groups of female HIV-seropositive participants as shown in Table 4. This is in agreement with the Cochrane meta-analysis by O'Brien et al. (2017) in which nineteen studies that involved progressive resistance exercise alone or combined with aerobic exercises. They found no significant difference in BMI of the exercising group compared with non-exercising control group. They reported clinically significant increase in body weight and girth with resistive exercise compared with nonexerciser.

This result is however in contrast with another systematic review that found a reduction in BMI when they examined the effect of a twice-weekly aerobic and progressive resistance exercise on body composition (Leach et al., 2015). The result was also not in line with the conclusion of Smith et al. (2001) who found that 12 weeks of supervised aerobic exercise training safely decreases fatigue, weight, BMI, subcutaneous fat and abdominal girth (central fat) in HIV-1-infected individuals. Fillipas et al. (2010) also found out in a systematic review that aerobic exercises decreases adiposity while progressive resistance exercise increases body weight, both of which affects the BMI. BMI is considered globally the most used adiposity index in the body composition area but it is an imperfect measurement of adiposity for not considering the composition and distribution of body fat (Beraldo et al., 2018). Thus, the lack of statistical significance could be attributed to an increase in muscle mass while there's a reduction in body fat.

The changes in body composition are training-specific, based upon the FITT training principle which varied from one study to another. Also, most studies reviewed worked on both male and female participants unlike this study that focused solely on female participants. Therefore, feminine factors such as weight distribution and hormonal fluctuations might have been of influence as the participants were mostly young adults and middle-aged women, a period during which hormonal surges are expected.

There was no statistically significant alteration in the waist to hip ratio of female HIVseropositive patients following 6-week aerobic and resistance training compared with nonexercising control group. These changes might not be statistically significant but they may be clinically significant as thus reduce the risk of several non-communicable diseases such as cardiovascular disease, diabetes mellitus and others.

O'Brien et al. (2017) found out in a Metaanalysis that there was no significant difference in the waist circumference of exercising HIV patients compared with non-exercising (control) HIV patients. Also in agreement with this study is a study carried out in Rwanda in which there was no significant difference in the waist circumference between HIV-seronegative and HIV-seropositive subjects following six months of combined exercise training (Mutimura et al., 2008). Leach et al. (2015) on the other hand found reduction in waist to hip ratio of HIV patients following twice-weekly aerobic and progressive resistance exercise. 
Several varying factors such as sample size, gender and duration of exercise training could be responsible for this divergent result. Most studies involved mainly male participants or combined male and female participants while this study focused solely on female participants. The duration of exercise training could play a role in the varying results since most studies ranged from six to twenty-four weeks while this study lasted for just six weeks in order to reduce the attrition rate.

Body fat percentage evaluated by BIA is considered the best double-indirect estimate of body fat and predictor of cardiovascular disease risk and presents a satisfactory performance but not exceeding the measures of central adiposity (Beraldo et al., 2018). The total and central adiposity as determined by body fat percentage and visceral fat of female HIV-seropositive patients did not significantly change following 6 weeks of combined aerobic and resistance training. In contrast to this study, Fillipas et al. (2010) found out in a systematic review that aerobic exercise decreases adiposity in HIV seropositive individuals. The duration and intensity of training could be responsible for this contrast as the participants in most of the studies reviewed trained for more than 6 weeks. Advanced stages of HIV infection and prolonged use of HAART especially Stavudine have been shown in previous studies to significantly affect adiposity and fat distribution in HIV patients. This study was delimited to HIV infections in clinical stages 1 and 2 . Thus, the effect of long duration of infection might not have played a significant role in this group of patients that participated in this study.

\section{Conclusion}

Based on the results that elicited from this study, it was concluded that six weeks of combined aerobic and resistance training exercise programme did not result in significant difference in the anthropometric parameters of BMI, percentage body fat, visceral fat and waist to hip ratio of female HIV-seropositive participants. It was therefore recommended that duration of exercise training is an important factor to be considered in designing exercise programme for female HIV-seropositive patients in order for them to get optimum health benefit. Also, supervised exercise training should be recommended for female HIV-seropositive patients in clinical stages 1 and 2 .

\section{Acknowledgement}

The authors wish to thank Mr. Omorotiomwan Imafidon and Mrs. Elizabeth Adams for their assistance in recruitment of participants and data collection.

\section{Funding}

There was no funding from any governmental or non-governmental organisation.

\section{Conflict of Interest}

There was no conflict of interest in carrying out this study.

\section{References}

American College of Sports Medicine. (2009). ACSM's Position Stand. Progression models in resistance training for healthy adults. Medical Science of Sports and Exercise, 41(3), 687-708.

American College of Sport Medicine. (2010). ACSM's guidelines for Exercise Testing and Prescriptions (8th Edition). Philadelphia (PA): Lippincot Williams \& Wilkins.

Beraldo, R.A., Meliscki, G.C., Silva, B.R., Navarro, A.M., Bollela, V.R., Schmidt, A. \& Foss-Freitas M.C. (2018). Anthropometric measures of central adiposity are highly concordant with predictors of cardiovascular disease risk in HIV patients. American Journal of Clinical Nutrition, 107, 883-893.

Bhattacherjee, A. (2012). Social science research: Principles, methods and practices. (2ndEdition). 
Textbooks Collection. Book 3. http://scholarcommons.usf.edu/oa_textbooks/3. Accessed on 23rd March, 2017.

Bokazhanova, A. \& Rutherford, G.W. (2006). The epidemiology of HIV and AIDS in the world. Coll Anthropology, 2, 3-10.

Centre for Disease Control. (2018). Guidelines for the Prevention and Treatment of Opportunistic Infections in Adults and Adolescents with HIV. Accessed from www.aidsinfo.nih.gov/guidelines on 8th August, 2019.

Crystal, S., Fleishman, J.A., Hays, R.D., Shapiro, M.F. \& Bozzette, S.A. (2000). Physical and role functioning among persons with HIV: results from a nationally representative survey. Sports Medicine, 38(12), 1210-1223.

Dudgeon, W.D., Phillips, K.D., Bopp, C.M. \& Hand, G.A. (2004). Physiological and Psychological Effects of Exercise Interventions in HIV disease. AIDS Patient Care and STDs, 18(2), 81-98.

Fillipas, S., Cherry, C.L., Cicutti, F., Smirneos, L. \& Holland, A.E. (2010). The effects of exercise training on metabolic and morphological outcomes for people living with HIV: A systematic review of randomised controlled trials. HIV Clinical Trials, 11(5), 270-282.

Gomes-Neto, M., Conceic, C.S., Carvalho, V.O. \& Brites, C. (2013). A systematic review of the effects of different types of therapeutic exercise on physiologic and fnctional measurements in patients with HIV/AIDS. Clinics, 68(8), 1157-1167.

Leach, L.L., Bassett, S.H., Smithdorf, G., Andrews, B.S. \& Travill, A.L. (2015). A Systematic Review of the Effects of Exercise Interventions on Body Composition in HIV+ Adults. Open AIDS Journal, 9, 66-79.

Mutimura, E., Crowther, N.J., Cade, T.W., Yarasheski, K.E. \& Steward, A. (2008). Exercise training reduces central adiposity and improves metabolic indices in HAART-treated HIV-positive subjects in Rwanda: A randomized controlled trial. AID Research and Human Retroviruses, 24(1), 15-23.

O’Brien, K.K., Tynan, A., Nixon, S.A. \& Glazier, R.H. (2016). Effectiveness of aerobic exercise for adults living with HIV: Systematic review and metaanalysis using the Cochrane Collaboration protocol. Biomedical Central Infectious Diseases, 16, 182.

World Health Organization (2017). Global Health Observatory (GHO) Prevalence of HIV among adults aged 15-49 (\%). Situation and trends. http://www.who.int/gho/hiv/epidemic_status/prev alence_text/en/. Accessed on $6^{\text {th }}$ March, 2017.

World Health Organization (2008). Waist circumference and waist to hip ratio: Report of WHO expert consultation. Geneva. 